\title{
Effects of water immersion on gait initiation: part II of a case series after incomplete spinal cord injury
}

\author{
Andresa R. Marinho-Buzelli $\mathbb{i}^{1} \cdot$ Ana Maria Forti Barela ${ }^{2}$ B. Catharine Craven $\mathbb{1}^{1,3} \cdot$ Kei Masani $\mathbb{( i )}^{1,4} \cdot$ \\ Hossein Rouhani ${ }^{5} \cdot$ Milos R. Popovic ${ }^{1,4} \cdot$ Mary C. Verrier ${ }^{1,6}$
}

Received: 1 June 2019 / Revised: 24 September 2019 / Accepted: 28 September 2019

(c) The Author(s), under exclusive licence to International Spinal Cord Society 2019

\begin{abstract}
Study design Case series.

Objectives This case series describes how the aquatic environment influences gait initiation in terms of the center of pressure (COP) excursion, impulses, trunk acceleration, and perceptions of participants with incomplete spinal cord injury (iSCI).

Setting Tertiary Rehabilitation Hospital, Ontario, Canada.

Methods Five individuals with iSCI (four cervical injuries/one thoracic injury, AIS D) participated in the study. Baseline clinical balance was evaluated by Berg Balance Scale and Mini-Balance Evaluation System Test. Participants initiated gait on a waterproof force plate and walked $\sim 4$ steps, in water and on land. COP trajectories during anticipatory and execution phases, impulses, and trunk acceleration parameters were investigated. Perceptions of walking in both environments were obtained using an interview.

Results COP trajectory was prominently longer when individuals stepped forward. A decrease in velocity of COP was observed predominantly in the AP direction during stepping. Non-normalized vertical impulses decreased as the AP impulses increased, in water compared to land. Upper to lower trunk acceleration ratios showed how water resistance influenced the lower trunk acceleration. Most of participants reported that walking in water was challenging, but safer than on land.

Conclusions Participants with higher balance function seemed to have more pronounced changes in anticipatory and execution phases' duration, length and velocity of COP. A faster anticipatory phase and a slower execution phase were observed in water than on land. Participants walked in water using a different trunk control strategy than on land and reported no fear of falling when walking in water versus land.
\end{abstract}

\section{Introduction}

Gait initiation is a transitory phase between standing posture and steady-state locomotion [1]. Although gait initiation has been investigated in populations with disabilities, such as in

Supplementary information The online version of this article (https:// doi.org/10.1038/s41394-019-0231-7) contains supplementary material, which is available to authorized users.

Andresa R. Marinho-Buzelli andresa.marinho@mail.utoronto.ca

1 KITE, Toronto Rehabilitation Institute, University Health Network, Toronto, ON, Canada

2 Institute of Physical Activity and Sport Sciences, Cruzeiro do Sul University, Sao Paulo, Brazil

3 Department of Medicine \& Rehabilitation Sciences Institute, individuals with Parkinson's disease and stroke, there are only a few studies describing some components of gait initiation after an incomplete spinal cord injury (iSCI) [2-4]. Postural stability during gait initiation has shown to be compromised in individuals with iSCI [4], deserving further investigation especially to inform application of therapeutic interventions.

The aquatic environment is often used to assist the first steps after a neurological injury, and has been shown to positively influence balance and walking functions in the

University of Toronto, Toronto, ON, Canada

4 Institute of Biomaterials and Biomedical Engineering, University of Toronto, Toronto, ON, Canada

5 Department of Mechanical Engineering, University of Alberta, Edmonton, AB, Canada

6 Department of Physical Therapy \& Rehabilitation Sciences Institute, University of Toronto, Toronto, ON, Canada 
early stages [5] and chronic stages [6] of locomotor recovery in individuals with neurological disorders other than iSCI. In the iSCI population, the evidence is scarce, although promising, suggesting that walking training in water could improve walking speed, distance and daily step activity [7]. The direct effects of immersion on steady-state walking have shown that walking in water is slower than walking on land $[8,9]$. We expected that the mechanical and thermal properties of water may also influence gait initiation performance compared to gait initiation on land, during anticipatory postural adjustment (APA) and execution phases of gait initiation. Therefore, we used a similar paradigm as in our previous studies $[10,11]$ with parameters of the center of pressure (COP) excursion, impulse forces, trunk acceleration, and reported perceptions during land and in-water performances to assist us in the understanding of different components of postural control when individuals with iSCI initiate gait in water in contrast to land.

Our previous paper, which is the first part of our study investigating the effects of water immersion on postural control in individuals with iSCI, suggested that the aquatic environment evokes postural disturbances during quasistatic standing without inducing the fear of falling [10]. In this second part of our study, we aim to explore for the first time the effects of water immersion during the transient phase to bipedal locomotion, gait initiation, across a series of case studies with individuals with iSCI with different motor and sensory functions.

\section{Methods}

\section{Participants and location}

Adult participants with iSCI were recruited through poster advertisement at a tertiary rehabilitation hospital during a 10-month period. Participants with a traumatic or nontraumatic etiology of iSCI who were able to initiate gait and walk at least three unassisted steps were included. Participants were excluded if they presented the following conditions: (i) history of mental, respiratory, cardiac or skin conditions that precluded immersion in warm water; (ii) participants who were unable to stand unsupported on land and in water; and (iii) individuals allergic to chlorine.

Based on these criteria, five adults with iSCI were eligible for participation (Table 1). Informed written consent was obtained from each participant. This study received approval from the research ethics board of the Toronto Rehabilitation Institute-University Health Network, Toronto, Canada (REB-10.029) in accordance with the declaration of Helsinki on the use of human participants in experiments.
Table 1 Participants' characteristics

\begin{tabular}{|c|c|c|c|c|c|}
\hline \multirow[t]{2}{*}{ Characteristics } & \multicolumn{5}{|c|}{ Participants } \\
\hline & 1 & 2 & 3 & 4 & 5 \\
\hline Age (years) & 61 & 69 & 42 & 53 & 56 \\
\hline Gender $(\mathrm{M} / \mathrm{F})$ & Male & Male & Male & Female & Male \\
\hline Height $(\mathrm{cm})$ & 177.8 & 174.0 & 180.3 & 157.5 & 180.3 \\
\hline Body weight on land $(N)$ & 784.6 & 568.7 & 720.9 & 484.8 & 676.3 \\
\hline Body weight in water $(N)$ & 480.1 & 324.0 & 417.3 & 182.8 & 355.9 \\
\hline$\%$ of body weight offloading & $38.8 \%$ & $43.0 \%$ & $42.1 \%$ & $62.3 \%$ & $47.4 \%$ \\
\hline Etiology of Impairment & $\begin{array}{l}\text { Non- } \\
\text { traumatic }\end{array}$ & Traumatic & $\begin{array}{l}\text { Non- } \\
\text { traumatic }\end{array}$ & Traumatic & Traumatic \\
\hline $\begin{array}{l}\text { Time since injury/surgery } \\
\text { (months) }\end{array}$ & 2 & 3 & 64 & 76 & 3 \\
\hline Neurological level & $\mathrm{C} 1$ & C6 & $\mathrm{T} 10$ & $\mathrm{C} 4$ & $\mathrm{C} 4$ \\
\hline AIS category & $\mathrm{D}$ & $\mathrm{D}$ & $\mathrm{D}$ & $\mathrm{D}$ & $\mathrm{D}$ \\
\hline $\begin{array}{l}\text { Upper limb motor score (Right/ } \\
\text { left }=25 / 25 \text { ) }\end{array}$ & $25 / 19$ & $21 / 21$ & $25 / 25$ & $22 / 25$ & $20 / 20$ \\
\hline $\begin{array}{l}\text { Lower limb motor score (Right/ } \\
\text { left }=25 / 25 \text { ) }\end{array}$ & $25 / 22$ & $25 / 22$ & $25 / 24$ & $24 / 24$ & $23 / 23$ \\
\hline Light touch $($ Right/left $=56 / 56)$ & $22 / 21$ & $54 / 49$ & $51 / 47$ & $46 / 47$ & $32 / 31$ \\
\hline Pin prick $($ Right $/$ left $=56 / 56)$ & $9 / 21$ & $56 / 56$ & $48 / 39$ & $36 / 37$ & $32 / 31$ \\
\hline Mobility function & Walking & Wheeling & Walking & Walking & Walking \\
\hline Assistive device for mobility & Cane & $\begin{array}{l}\text { Power } \\
\text { wheelchair }\end{array}$ & Cane & None & Rollator \\
\hline
\end{tabular}

AIS American Spinal Injury Association Impairment Scale 


\section{Clinical examination}

The clinical assessment prior to tests in the aquatic and land environments included the assessment of participants' neurological impairment through the International Standards for Neurological Classification of Spinal Cord injury (ISNCSCI) [12, 13], and the clinical balance function assessed through the Berg Balance Scale (BBS) [14] and the Mini-Balance Evaluation System Test (Mini-BESTest) [15] (Table 2). Details of the clinical examination are reported in our previous study [10].

\section{Instrumentation}

A therapeutic pool measuring $9.8 \times 4.9 \times 1.1 \mathrm{~m}$ with temperature set at 34-35 degrees Celsius, was used for the assessment of gait initiation in the water. The area outside the therapy pool was used for the assessment of gait initiation on land. In both settings (i.e., land and water), we used the same instrumentation as described in our prior study with able-bodied subjects [11] and with individuals with iSCI [10], which included a waterproof force plate (AMTI, ORP-WP-1000, USA) and three wireless bodyworn inertial sensors (Physilog, BioAGM, Switzerland) sealed in waterproof bags (aLoksak, Inc., Naples, USA). The inertial sensors were attached to the upper trunk region (head of sternum), and to the lower trunk region overlying L5/S1 landmarks. Data from the force plate (sampled at $1000 \mathrm{~Hz}$ ) and from the inertial sensors (sampled at $500 \mathrm{~Hz}$ ) were synchronized and analyzed using custom-developed routines on MATLAB (Mathworks Inc., Natick, USA).

\section{Experimental procedures}

Participants performed gait initiation on land and in water in 2 consecutive days. Exception was Participant 3 who performed one experiment on day 1 and the other one on day 3 due to conflict in his schedule.

Prior to starting to walk, participants stood in the same comfortable standing position. Participants were instructed to initiate gait using their preferred leg, immediately after a visual command. The locations of feet were marked on the force plate with water-resistant chalk to maintain the exact same feet locations between the conditions and among trials. A light was positioned at eye level and 3.5-m distance and was activated following 5 to $10 \mathrm{~s}$ of quiet standing. Participants walked $\sim 4$ steps with their upper limbs positioned above the water surface to avoid additional water resistance beyond what was delivered to the lower body. Ten trials of gait initiation were performed on land and ten trials were performed in water.

After completion of tests on land and in water, all participants responded to an interview consisting of five
Table 2 Classification of participants' clinical balance

\begin{tabular}{llllll}
\hline Balance scales & \multicolumn{3}{l}{ Participants } \\
\cline { 2 - 6 } & 1 & 2 & 3 & 4 & 5 \\
\hline Mini-BESTest & 2 & 2 & 4 & 5 & 4 \\
$\quad$ Anticipatory (0-6) & 1 & 1 & 3 & 5 & 6 \\
Reactive postural control (0-6) & 5 & 4 & 5 & 6 & 6 \\
Sensory orientation (0-6) & 8 & 1 & 7 & 8 & 10 \\
$\quad$ Dynamic gait (0-10) & 16 & 8 & 19 & 24 & 26 \\
Mini-BESTest (total: 0-28) & 51 & 42 & 45 & 55 & 56 \\
Berg Balance Scale (total: 0-56) & & & & & \\
\hline
\end{tabular}

questions investigating their perceptions of walking in water and on land (Supplementary information).

\section{Data analysis}

We calculated the percentage of body weight (BW) offloading in water using the equation below:

$\%$ offloading $=100 \times\left(\mathrm{BW}_{\text {land }}-\mathrm{BW}_{\text {water }}\right) / \mathrm{BW}_{\text {land }}$

where: $\mathrm{BW}$ on dry land $\left(\mathrm{BW}_{\text {land }}\right)$ and apparent $\mathrm{BW}$ in water $\left(\mathrm{BW}_{\text {water }}\right)$ were computed from the vertical component of ground reaction force (data acquired with the force plate) during the last $2 \mathrm{~s}$ of quiet standing prior to initiating walking.

Butterworth low-pass filters were used for the force plate signals (second order with a cut-off frequency of $10 \mathrm{~Hz}$ ) and inertial sensors signals (fourth-order with a cut-off frequency of $30 \mathrm{~Hz}$ ) [11].

As in our previous study [11], gait initiation phase was defined from the first dynamic event on the force plate $\left(\mathrm{APA}_{\mathrm{ONSET}}\right)$ to heel-strike of the first swing limb, determined through the free vertical moment on the force plate (Fig. 1) $[11,16]$. We used the $\mathrm{APA}_{\mathrm{END}}$ point to mark the beginning of the execution phase on the ground reaction force (GRF) and acceleration signals.

We summarized the dependent variables of all trials in each condition in mean $\pm \mathrm{SD}$. We used a thematic analysis to identify the themes from the participants narrative as per Braun and Clarke [17]. One researcher (AMB) searched, identified, and developed initial themes. A second member of the research team (MCV) reviewed the themes to ensure they were representative of the data and to ensure theme saturation.

\section{Dependent variables}

We calculated the duration $(\mathrm{s})$, length $(\mathrm{cm})$, and velocity $(\mathrm{cm} / \mathrm{s})$ of the COP within the APA, EXE1, and EXE2 trajectories. The impulse generated during the execution 


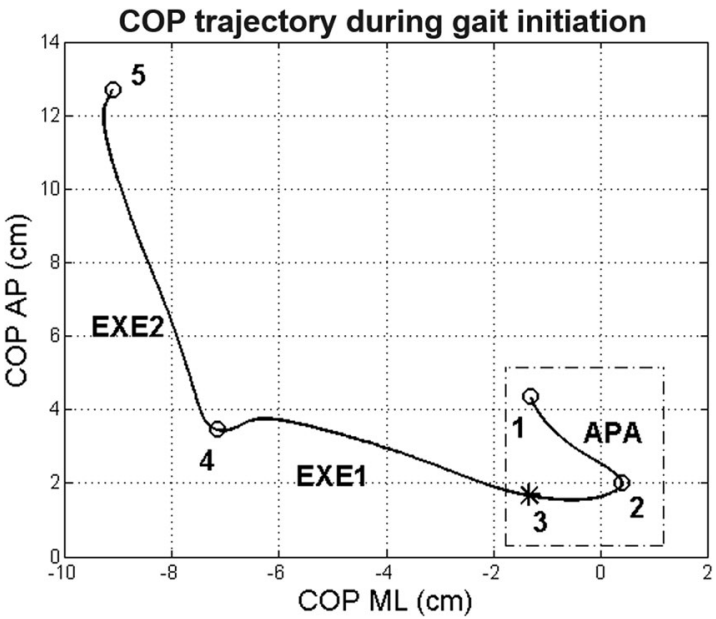

Fig. 1 Center of pressure (COP) horizontal trajectory during gait initiation with the right leg on land. (1) Onset of Anticipatory Postural Adjustment (APA) phase. (2) Peak of APA phase. (3) End of APA phase (beginning of Execution phase). (4) Most lateral and posterior landmark towards the first stance limb. (5) End of gait initiation phase detected on the negative peak of the free vertical moment (FVM). APA phase was defined from the event 1 to the event 3. Execution phase 1 (EXE1) was defined from the event 3 to the event 4, and Execution phase 2 (EXE2) was defined from the event 4 to the event 5

period of gait initiation was calculated by integrating the area under the curves of anteroposterior (AP) and vertical forces. Duration (s) of impulse, impulse forces normalized by body weight (BW) (\%BW.s) and non-normalized by BW (N.s), and forces $(N)$ in AP and vertical directions were calculated during the execution period of gait initiation (i.e., from $\mathrm{APA}_{\mathrm{END}}$ to heel-strike).

We calculated the root mean square (RMS) acceleration (in $\mathrm{m} / \mathrm{s}^{2}$ ) of the upper and lower trunk in the AP and mediolateral (ML) directions during Execution. The Execution was from the end of APA (APA $\mathrm{END}_{\mathrm{E}}$ ) to the end of gait initiation phase. In order to analyze how upper and lower trunk accelerations changed in relation to each other, we calculated the upper trunk to lower trunk acceleration ratio in AP and ML directions during Execution phase.

\section{Results}

\section{Clinical balance}

The entire classification of clinical balance is presented in Table 2. The BBS score varied from 42 to 56 among participants. Participants 4 and 5 presented the highest balance performance for the BBS and Mini-BESTest. Participant 2 presented the lowest balance score both on the BBS and the Mini-BESTest and was the one using a power wheelchair to assist his mobility.

\section{Gait initiation biomechanical parameters}

\section{COP parameters}

Figure 2 illustrates the COP trajectory during gait initiation on land and in water from two participants, one with a moderate light touch and pin prick deficits and relatively low-balance function on the Mini-BESTest (P1), and the other with a high-balance function (P4) (Table 3). Overall, Participant 1 presented more inflection points in the COP trajectory in water and on land during the execution of gait initiation in contrast to the COP trajectory with less inflection points of the Participant 4. In addition, Participant 4 presented a larger APA in water (AP-water: 6.02 \pm $1.42 \mathrm{~cm}$ ) compared to dry land (AP-land: $2.22 \pm 1.09 \mathrm{~cm}$ ), while Participant 1 did not respond with a larger APA in water.

Table 3 describes the COP parameters during APA, EXE1 and EXE2, separately. During APA, the length and velocity in AP and ML directions increased in water in contrast to land, more accentuated in the participants who presented higher balance function. For example, APA length and duration increased in Participants 4 and 5, only.

During the weight transfer phase, i.e., the COP EXE1, the responses were more inconsistent across participants. The ML COP length was decreased more accentuated in Participant 2 (land: $13.69 \pm 2.10$, water: $7.14 \pm 1.50$ ), who had the lowest balance score. Participant 4, with the shortest height $(157.5 \mathrm{~cm})$ and higher body offloading (62.3\%), presented a much slower velocity in AP (land: $10.39 \pm 2.16$, water: $5.86 \pm 2.78$ ) and ML direction (land: $23.25 \pm 2.17$, water: $16.45 \pm 5.31$ ) in water during EXE1 (Table 3).

The pronounced changes in COP trajectory occurred in EXE2 phase, i.e., the COP trajectory occurring while individuals were stepping forward, from approximate toe-off to the heel-strike of the swing limb (Fig. 2). The duration and length of EXE2 in AP and ML directions were longer in water than on land across all participants. A decrease in COP velocity in AP direction was observed in water in contrast to land, more evident in Participant 4.

\section{Impulses}

All participants presented larger impulse durations during the execution phase of gait initiation while stepping in water compared to on land (Table 4). The vertical and AP impulses, normalized by body weight in each environment, increased in water compared to on land. The impulse not normalized by the body weight slightly reduced in vertical direction in most participants and increased in AP direction in water when contrasted to land. 


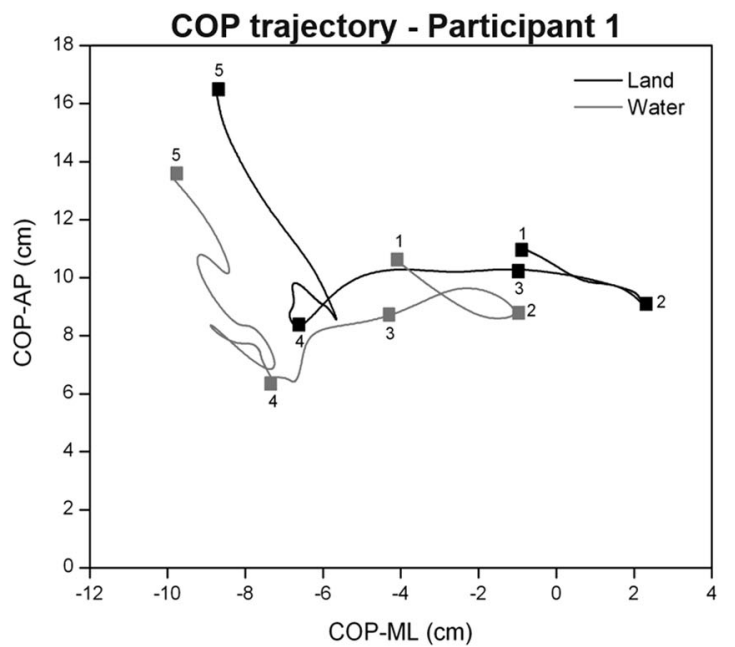

Fig. 2 Center of pressure (COP) horizontal trajectories during gait initiation with the right leg, on land (black trace) and in water (gray trace). Left: COP trajectory of a participant with low-functioning balance performance (Participant 1). Right: COP trajectory of a participant with high-functioning balance performance (Participant 4).

\section{Trunk acceleration parameters}

Table 5 shows the results of RMS acceleration of upper and lower trunk in AP and ML directions during execution of the first step. The acceleration of upper trunk in the ML direction, along with the lower trunk in the AP and ML directions presented a more accentuated decrease in water, in contrast to the acceleration on land, across all participants, except for Participant 4 who increased upper trunk acceleration in the AP direction. When examining the upper trunk to lower trunk acceleration ratios, most participants had a noticeable increased ratio in the AP direction, where Participant 4 had the most accentuated upper/lower trunk ratio increase in water in contrast to land and in contrast to other participants (Fig. 3).

\section{Participants perceptions while walking in water and on land}

Table 6 displays the supporting quotes from participants on the following three main themes analyzed across the dataset: (1) The aquatic environment as a facilitator for walking function, (2) Challenges while walking in water and on land, and (3) Safety.

\section{Discussion}

This study is the first to describe gait initiation in water after iSCI, measured by parameters of COP trajectory, impulse forces, trunk acceleration, and individual perceptions. The different neurological and balance control attributes of

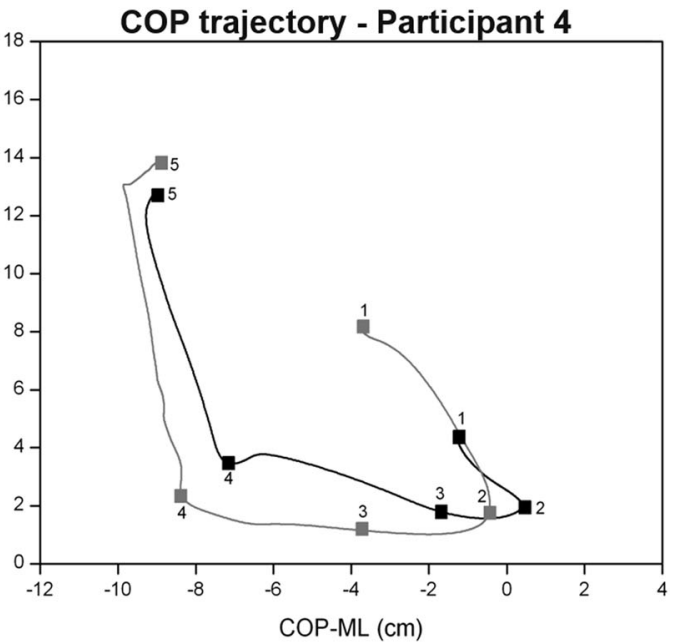

The COP trajectory is representative of a single trial. APA: from COP onset (1) to the end of APA (3). COP EXE1 from the end of APA (3) to the approximate toe-off of swing limb (4). COP EXE2 from toe-off (4) to the end of COP trajectory (5). (2) APA peak in mediolateral direction

participants helped to explore whether the aquatic environment could influence both the quantitative parameters and qualitative perceptions of walking in water depending on each participant's ability and interactions with the aquatic and land environments. The aquatic environment influenced the performance of gait initiation in all five participants during anticipatory and execution phases, providing both challenges and support while participants initiated gait in water.

Through the COP trajectory, we explored how the aquatic environment influenced the anticipatory and execution phases of gait initiation of individuals with different neurological deficits. For example, Participant 1, who presented with a substantial sensory deficit aligned with a moderate deficit in anticipatory and reactive balance control, was the only individual who demonstrated decreases in APA length in water in comparison to land. In contrast, the participants with higher balance function (P4 and P5) were those with the most moderate increase in APA length and AP velocity in the aquatic environment. In four participants, the length of COP trajectory in AP direction increased substantially during EXE2 while the velocity of the COP trajectory in AP direction decreased. Our previous studies with able-bodied participants showed similar results, i.e., longer COP trajectory with decreased COP velocity when starting to walk in water compared to dry land, potentially due to the effect of the water resistance on the lower body $[11,18]$.

All participants had greater AP impulses while executing the first step in water than on land. In previous studies examining impulses during the steady-state gait cycle in young [19] and elderly [20] participants, the horizontal 


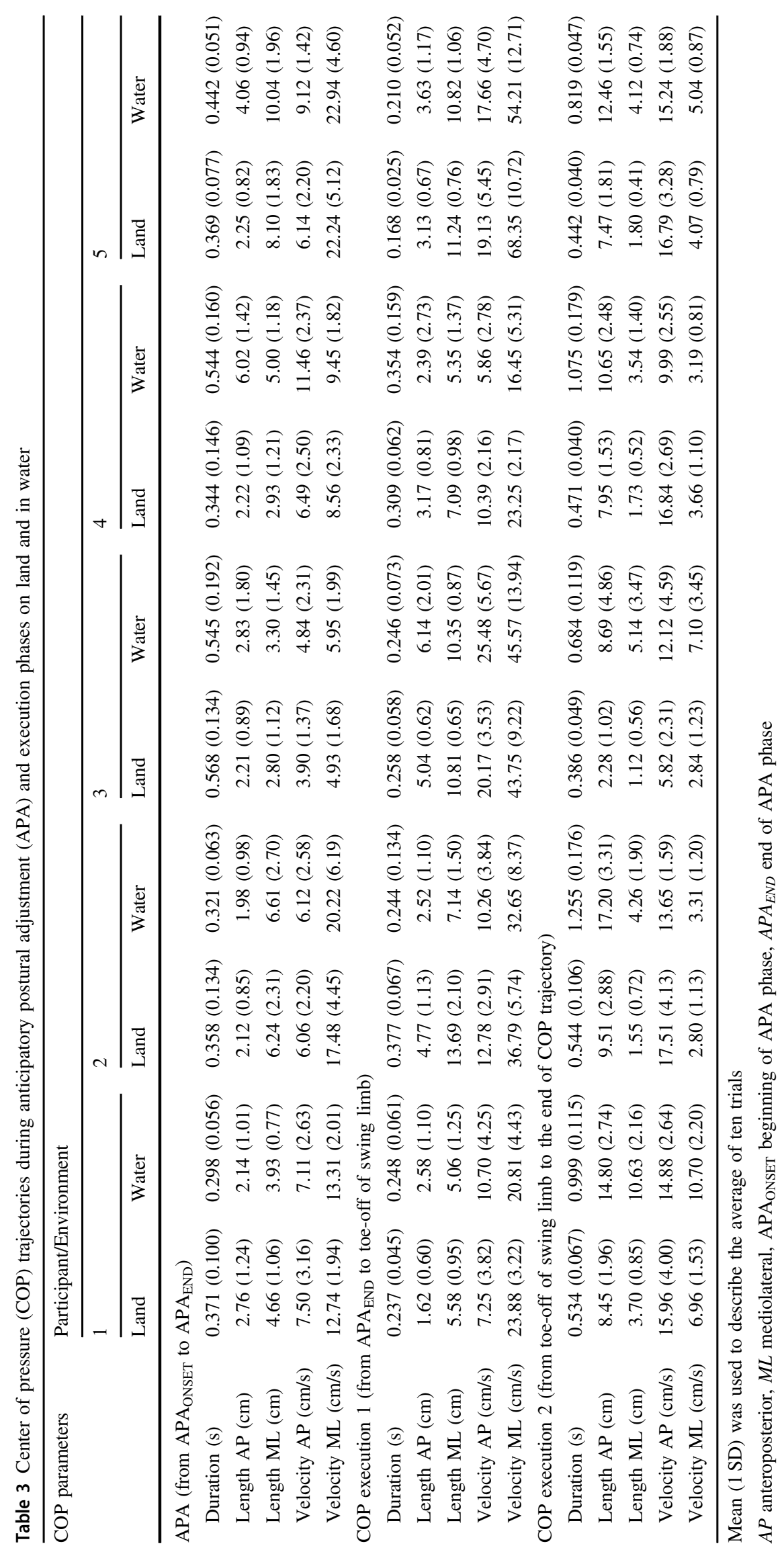




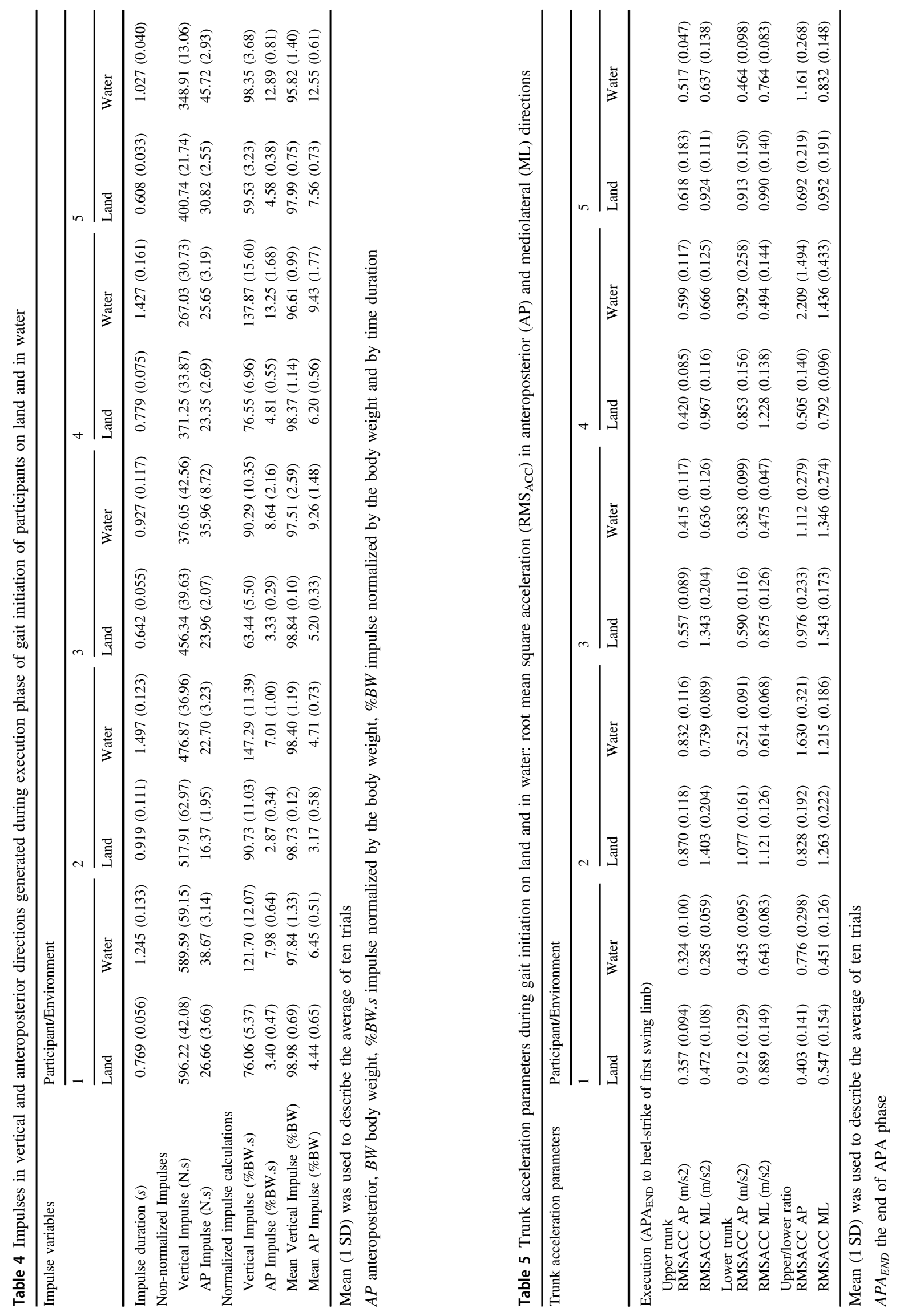


Fig. 3 Upper to lower trunk acceleration (ACC) ratio in anteroposterior (AP) direction of all five participants during ten trials of gait initiation in water (gray line) and on land (black line)

\section{Upper to lower trunk acceleration (ACC) ratio in AP direction}
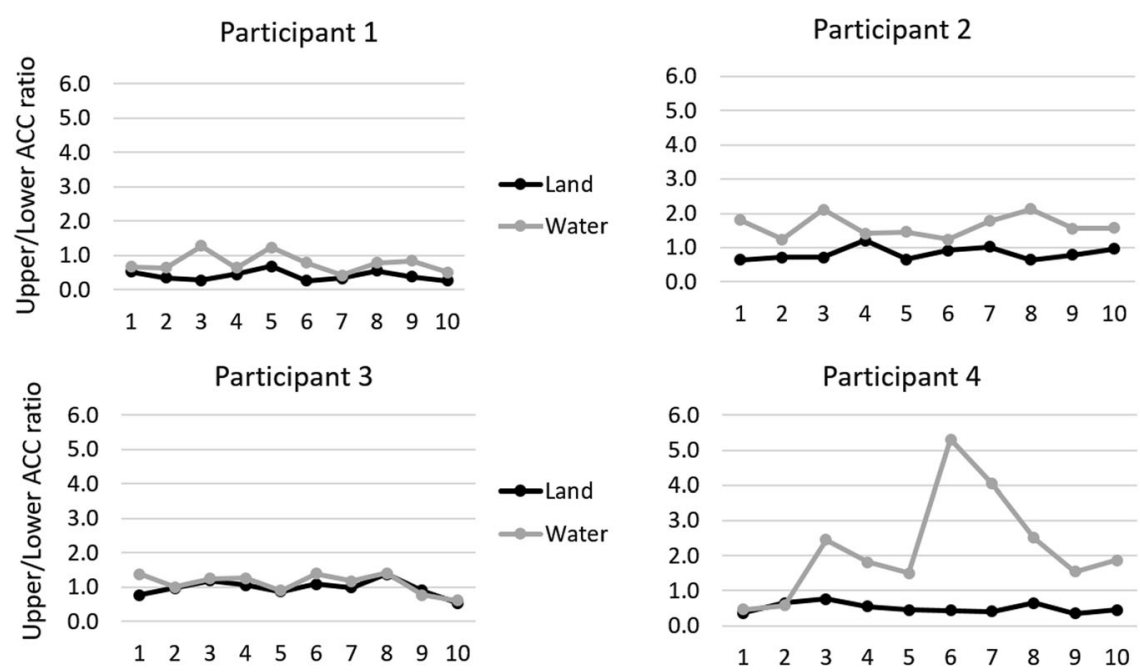

Participant 5

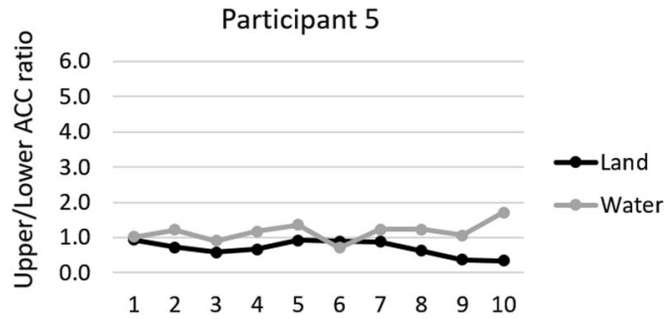

impulses were higher in water than on land for both the groups. In the present study, the analysis of the nonnormalized impulse and the mean impulse force showed a decrease in the vertical impulse in water versus the land, suggesting the impact of buoyancy of the water while initiating gait.

The RMS acceleration of upper and lower trunk detected different trunk acceleration patterns to maintain balance while initiating gait in water compared to land. There was an increase in RMS acceleration ratio (upper trunk/lower trunk) in the AP direction in water compared to land in most participants. As the participants walked forward in water when immersed to the umbilicus level, the water resistance applied to the lower body decreased acceleration in the lower trunk, while the upper trunk moved forward freely. The resistance on the lower body, aligned with the buoyancy of the water, appears to be an incentive for a new trunk strategy to maintain stability in water and seems to evoke a different pattern of trunk control to maintain balance while initiating gait. A change in the trunk strategy in water could be incorporated into training programs for enhancing balance control that could be beneficial for dynamic activities of daily living during standing.

The participants' perceptions supported our interpretation of quantitative parameters of gait initiation in water and on land. For example, most participants reported the slowing down of walking, suggesting that the water resistance was a force opposing gait initiation. In fact, both the COP and GRF parameters showed more clearly the influence of resistance on decreasing velocity of COP and increasing the AP mean force while participants walked forward. While the resistance caused by water challenged the participants, the buoyancy and hydrostatic pressure may have supported the participants' during the step in the execution phase. Most patients reported that they felt secure and safe to step forward surrounded by water. Four of five participants reported that they did not have to worry about falling because of the perceived support from the water pressure.

Other relevant clinical characteristics, such as trunk control, somatotype, and the proportion of lean and fat mass of participants, could potentially add to the interpretation of the quantitative and qualitative measures of gait initiation performance, both in water and on land. Particularly, if a more representative sample of participants with iSCI with stratification based on differing degrees of trunk control could be studied.

The present study complements our previous study investigating the effects of the aquatic environment on quasi-static standing posture [10]. Our findings indicate that the aquatic medium also influences the dynamic postural control during gait initiation and suggests that immersion in water prolonged the execution of gait initiation and may facilitate longer step execution during training in the early stages of SCI. We suggest that the increasing resistance to 
Table 6 Themes and supporting quotes from participants

Theme 1: the aquatic environment as a facilitator for walking function

"In water, I felt I was walking in a straighter line versus dry land. I felt like I had more support in water." (Participant 1)

"I would walk and swing without losing my balance in water."

(Participant 2)

"Much easier and effortless in water. I felt my posture is better too. I am walking [in water] I feel I have more control on my legs... In water, I'm more confident." (Participant 3)

"In water, you can kind of let the forces around to help. You can take it easy." (Participant 4)

"The water is very helpful. First I came here [at the rehab center] was on land. When I came to water, I could start walking. After the water, I could get more control of my body. Just one session! Before, this session in water, 2 or 3 people were walking me. After the water, I could walk myself alone." (Participant 5 )

Theme 2: challenges while walking in water and on land

"Walking was steady but was more difficult because of the force and weight of the water." (Participant 1)

"You can't walk as fast [in water]." (Participant 2)

"On land, I have this insecurity I am going to fall." (Participant 3)

"When I walk on land, was all jerky... In water, I did more side to side. I know I swayed more" (Participant 4)

"In the water, I should have to pay attention more. My legs are lighter and I have to push it more." (Participant 5)

"Walking is harder in water, because I have resistance."

(Participant 5)

Theme 3: safety

"It was harder to walk in water because of the resistance, but I felt more secure in water... There was water pressure around me."

(Participant 2)

"It is easier to do activities in water. Don't have to worry about falling or breaking something."(Participant 3)

"I walked better on land, but the consequences of falling is greater on land." (Participant 4)

"I can walk more confident inside water, because something is holding me." (Participant 5)

the lower limbs and the challenges of the trunk movement patterns during step initiation in the aquatic environment may be a coordinated training strategy worth pursuing to augment therapeutic outcomes. Altering the velocity of movement performed and the level of immersion in water provides an approach to change the water resistance and buoyancy levels incrementally. The versatility of the aquatic environment could be a venue for training specificity for individuals with different sensorimotor and balance dysfunctions.

Future studies should explore further paradigms of postural control incorporating both quiet standing posture and gait initiation, as well as patients' perceptions when investigating how the application of customized therapeutic interventions, such as aquatic therapy, might contribute to improvement of postural control in individuals with varying sensorimotor deficits in both traumatic and nontraumatic iSCI.
Acknowledgements Special thanks to the physiotherapist Mr. Chris Allapat for assessing the clinical balance and neurological functions of study participants. We thank Dr. Kamiar Aminian for providing the inertial sensor system. We also acknowledge the research and technical support of Adolazim Rashidi and Carlos Buzelli Neto during the experiments, and the assistance of the summer students from the SCI Mobility Laboratory at Toronto Rehabilitation Institute-University Health Network.

Funding Dr. Marinho-Buzelli acknowledges the support of Canadian Institutes of Health and Research (CIHR) through the Vanier Canada Graduate Scholarship on the development of this study [Grant \# 95662]. Dr. Rouhani acknowledges the support of the Swiss National Science Foundation Grants [PBELP3-137539 and P300P2-147865] and Spinal Cord Injury Ontario Postdoctoral Fellowship. Dr. Craven acknowledges support from the Toronto Rehab Foundation as the Toronto Rehab Chair in SCI Rehabilitation. This research was also supported by the Natural Sciences and Engineering Research Council grant [\#249669], Ontario Neurotrauma Foundation, Réseau Provincial de Recherche en Adaptation-Réadaptation (REPAR) and KITE, Toronto Rehab-University Health Network.

\section{Compliance with ethical standards}

Conflict of interest The authors declare that they have no conflict of interest.

Publisher's note Springer Nature remains neutral with regard to jurisdictional claims in published maps and institutional affiliations.

\section{References}

1. Winter D. Human balance and posture control during standing and walking. Gait Posture [Internet]. 1995;3:193-214. Available from http://linkinghub.elsevier.com/retrieve/pii/0966636296828499.

2. Chang H, Chuang T, Lee S, Liao S, Lee H, Shih Y. et al. Temporal differences in relative phasing of gait initiation and first step length in patients with cervical and lumbosacral spinal cord injuries. Spinal Cord [Internet]. 2004;42:281-9. Available from http://www.nature.com/doifinder/10.1038/sj.sc.3101587.

3. Dutta A, Kobetic R, Triolo RJ. Gait initiation with electromyographically triggered electrical stimulation in people with partial paralysis. J Biomech Eng [Internet]. 2009;131:1-9. Available from http://biomechanical.asmedigitalcollection.asme. org/article.aspx ?articleid $=1475745 \% 5 \mathrm{Cn}$, http://search.ebscohost. com/login.aspx ?direct $=$ true $\& \mathrm{db}=\mathrm{cmedm} \& \mathrm{AN}=$ $19604014 \&$ site $=$ ehost-live

4. Lemay J-F, Duclos C, Nadeau S, Gagnon DH. Postural control during gait initiation and termination of adults with incomplete spinal cord injury. Hum Mov Sci [Internet]. 2015;41:20-31. Available from http://www.sciencedirect.com/science/article/pii/ S0167945715000226\%5Cn, http://ac.els-cdn.com/ S0167945715000226/1-s2.0-S0167945715000226-main.pdf? _tid=25b1303a-6ddd-11e5-9b65-00000aab0f6c\&acdnat = 1444323409 c80b402a85d77c08a26e77d19aa7fc46.

5. Tripp F, Krakow K. Effects of an aquatic therapy approach (Halliwick-Therapy) on functional mobility in subacute stroke patients: a randomized controlled trial. Clin Rehabil. 2014;28:432-9.

6. Marinho-Buzelli AR, Bonnyman AM, Verrier MC. The effects of aquatic therapy on mobility of individuals with neurological diseases: a systematic review. Clin Rehabil [Internet]. 2015;29:741-51. Available from: http://journals.sagepub.com/doi/ $10.1177 / 0269215514556297$. 
7. Stevens SL, Caputo JL, Fuller DK, Morgan DW. Effects of underwater treadmill training on leg strength, balance, and walking performance in adults with incomplete spinal cord injury. J Spinal Cord Med [Internet]. 2015;38:91-101. Available from http://www. tandfonline.com/doi/full/10.1179/2045772314Y.0000000217.

8. Tamburella F, Scivoletto G, Cosentino E, Molinari M. Walking in water and on land after an incomplete spinal cord injury. Am J Phys Med Med [Internet]. 2013;92:e4-15. Available from http:// link.springer.com/chapter/10.1007/978-3-642-34546-3_119.

9. Iucksch DD, Israel VL, Ribas DIR, Manffra EF. Gait characteristics of persons with incomplete spinal cord injury in shallow water. J Rehabil Med. 2013;45:860-5.

10. Marinho-Buzelli AR, Rouhani H, Craven BC, Masani K, Barela JA, Popovic MR, et al. Effects of water immersion on quasi-static standing exploring center of pressure sway and trunk acceleration: a case series after incomplete spinal cord injury. Spinal Cord Ser Cases [Internet]. 2019;5:5. Available from https://doi.org/10. 1038/s41394-019-0147-2. eCollection 2019.

11. Marinho-Buzelli AR, Masani K, Rouhani H, Barela AM, Fernandes GTB, Verrier MC, et al. The influence of the aquatic environment on the center of pressure, impulses and upper and lower trunk accelerations during gait initiation. Gait Posture. 2017;58:469-75.

12. Kirshblum SC, Waring W, Biering-Sorensen F, Burns SP, Johansen M, Schmidt-Read M. et al. International standards for neurological classification of spinal cord injury. J Spinal Cord Med [Internet]. 2011;34:535-46. Available from http://www.ta ndfonline.com/doi/full/10.1179/107902611X13186000420242.
13. Walden K, Bélanger LM, Biering-Sørensen F, Burns SP, Echeverria E, Kirshblum S. et al. Development and validation of a computerized algorithm for International Standards for Neurological Classification of Spinal Cord Injury (ISNCSCI). Spinal Cord [Internet]. 2016;54:197-203. Available from http://www.ncbi. nlm.nih.gov/pubmed/26323348.

14. Berg K. Measuring balance in the elderly: Development and validation of an instrument. Can J Public Heal J Public Heal. 1992;83:S7-11.

15. Franchignoni F, Horak F, Godi M, Nardone A, Giordano A. Using psychometric techniques to improve the balance evaluation systems test: The Mini-BESTest. J Rehabil Med. 2010;42:323-31.

16. Moineau B, Boisgontier MP, Barbieri G, Nougier V. A new method to assess temporal features of gait initiation with a single force plate. Gait Posture [Internet]. 2014;39:631-3. https://doi.org/ 10.1016/j.gaitpost.2013.07.007.

17. Braun V, Clarke V. Using thematic analysis in psychology. Qual Res Psychol. 2006;3:77-101.

18. Marinho-Buzelli AR, Barela AMF, Barela JA, Celestino ML, Popovic MR, Verrier M. The influence of the aquatic environment on gait initiation: a pilot study. Motor Control. 2017;21:211-26. https://doi.org/10.1123/mc.2015-0091.

19. Barela AMF, Stolf SF, Duarte M. Biomechanical characteristics of adults walking in shallow water and on land. J Electromyogr Kinesiol. 2006;16:250-6.

20. Barela AMF, Duarte M. Biomechanical characteristics of elderly individuals walking on land and in water. J Electromyogr Kinesiol. 2008;18:446-54. 